Témoigner

Getuigen
Témoigner. Entre histoire et mémoire

Revue pluridisciplinaire de la Fondation Auschwitz

$118 \mid 2014$

Au nom des victimes. Dictature et terreur d'État en Argentine, Chili et Uruguay

\title{
Quand la victime est introuvable
}

À propos des détenus-disparus au Chili et des parents qui les ont cherchés pendant et après la dictature (1973-1989)

When the victim is nowhere to be found. Chile's "disappeared" and the families that have been searching for them during and after the dictatorship (1973-1989) Wat als de slachtoffers spoorloos zijn? De zoektocht naar verdwenen gevangenen tijdens en na de dictatuur (1973-1989)

\section{Antonia García Castro}

Traducteur : Antonia García Castro

\section{(2) OpenEdition}

\section{Journals}

Édition électronique

URL : http://journals.openedition.org/temoigner/922

DOI : 10.4000/temoigner.922

ISSN : 2506-6390

Éditeur :

Éditions du Centre d'études et de documentation Mémoire d'Auschwitz, Éditions Kimé

Édition imprimée

Date de publication : 1 octobre 2014

Pagination : 87-95

ISBN : 978-2-84174-674-3

ISSN : 2031-4183

Référence électronique

Antonia García Castro, «Quand la victime est introuvable», Témoigner. Entre histoire et mémoire [En ligne], 118 | 2014, mis en ligne le 01 octobre 2015, consulté le 23 octobre 2020. URL : http:// journals.openedition.org/temoigner/922 ; DOI : https://doi.org/10.4000/temoigner.922 


\section{CHIL}

\section{Quand la victime est introuvable}

À propos des détenus-disparus au Chili

et des parents qui les ont cherchés pendant

et après la dictature (1973-1989)

$\rightarrow \operatorname{Par}$ Antonia García

Castro, Radio Universidad

de Chile d'entrée de jeu qui sera, aux mains des militaires, plus qu'un moyen d'éliminer des opposants : une arme de contrôle de vastes couches de la population désorientée et démobilisées par l'absence d'informations sur le sort de certains prisonniers.

Au-delà du cas chilien, on peut rappeler qu'en Amérique latine, la disparition devient une pratique coercitive de l'État face à la remise en question des privilèges de certains secteurs sociaux et l'irruption sur la scène politique d'autres secteurs auparavant exclus. Dans ce contexte régional de redistribution des rôles, aux configurations diverses selon les pays, va se mettre en place une coopération internationale destinée à dissuader de toute contestation. Elle consiste notamment dans l'exportationdes savoirs en matière de lutte antisubversive dontla disparition forcé est instruments 1 .

Par rapport à ces logiques, la disparition a toujours au moins deux cibles: celui qui disparaît et celui qui est susceptible de subir les effets de cette disparition. Sans nier cette dimension, sans nier leur propre douleur, les familles organisées en association vont manier le terme de «victime » avec pudeur et d'une manière sensiblement différente selon les périodes. Dans le cadre de cet article ${ }^{2}$, on se limitera à considérer certains usages exemplaires tout en rendant compte d'un type de raisonnement ic décliné en quatre temps : à travers l'examen des logiques politiques à l'œuvre ; en procédant à une caractérisation de l'AFDD ; en examinant le besoin de mettre en mots ; enfin en abordant quelques dilemmes auxquels les familles ontété confrontées plus récemmentsols des gouvernenents démocratiques. Celanis cés plénè aussi ce qu'elles ont refusé d'en faire. 
CHILI
Quand la victime Quand la victime
est introuvable

L'histoire de l'AFDD est l'histoire d'une prise de conscience. C'est en menan une recherche au nom d'un ou de plusieurs parents que les familles vont se rendre compte de la situation que connaissent les leurs. Ce qu'elles comprendront, elles le communiqueront de maintes manières à l'ensemble de la société. En parallèle, elles consigneront un grand nombre de documents destinés à permettre des démarches légales auprès de diverses autorités. Ce faisant, elles vont constituer des archives d' d'une grande valeur. En effet, il n'est pas exagére de dire que tout ce que l'on sai aujourd'hui sur les détenus-disparus ${ }^{3}$ et sur les disparitions forcées au Chili, on le doit d'abord aux familles et à l'organisme qui leur a permis de s'organiser : le Vicariat de la solidaritét

Ce travail de documentation est le moins connu de tous : sorte de coulisse de la (3) Léxpression utilisée au Chili Dàs 1973 |'Archevêâue de Santiago avait créé un ur organisme dont la mission principale
était de porter assistance personnes poursuivives pour des motiff politiques. La junte dissoudre cette structure, celues'était exécuté, mais avait créé dès le lendemain, en janvier 1975 même mission. Le Vicariat de solidarité sera toléré - non sans
leprésailles !- tout au long de la dictature.

(5) Cf. La Spirale, documentaire Meppiel et Valérie Mayoux (1976).

(6) Sur cette dimension : Les travaux menés par la revue des ennées 1990 , en particulies: "Les Disparitions", Cultures $\&$ Conflits, , $\circ^{\circ} 13$ et 11.
L'Harmattan, 1994.

(7) En espagnol «familiares »: femmes, des mères, mais aussi des épouses, des soeurs, des parents n'ont pas nécessairement
rejoint l'Association. Quand on parle dans ce texte des familles on sous-entend « "les fan
membres de l'AFDD \%. (8) L'Association des familles
des exécutés politiques. Puis les diverses associations de familles 作 les familles vont appréhender est la dimension politique du crime. Et pour cause les arrestations dont elles ont souvent été témoins sont contemporaines du coup d'Etat de 1973. Pendant les premiers jours, on assiste à une sorte de représentation apocalyptique du retourà àlordre faisant suite à trois ans de gouvernement socialiste. Un gouvenemt qui a littér droites chiliennes (a) Palais présidentiel, des arrestations massives ont lieu, on convertit des stades en prisons. Mais le caractère massif de la répression ne signifie pas qu'elle s'exerçait arbitrairement. Les victimes n'ont pas été choisies au hasard.

L'identité des prisonniers est l'un des éléments clés pour comprendre les logiques politiques. Notamment l'engagement (le fait d'être membre d'un parti, d'un syndicat, d'une association), les professions exercées ainsi que l'appartenance à certains secteurs sociaux. Car il y a eu des îlots protégés pendant la dictature. Il y eut très nettement des quartiers intouchables, sauf cas exceptionnels, et des familles qui ne pouvaient quêtre àl'abri puisqu'elles avaient approuvé le coup d'État En revanche, des zones populaires connues pour l'adhésion de leurs habitants au gouvernement l'Unitépoplaire (1970-1973) ntétésystén

Ces éléments n'épuisent pas les logiques politiques que la disparition met en œuvre ${ }^{6}$. Mais dans les discours et les pratiques des familles, cette dimension vajouer un rôle central. Aux yeux de celles-ci, les détenus-disparus seront principalement des acteurs sociaux et politiques qui, en tant que tels, ont été victimes d'un crime particulier

\section{AGIR AUNOM D'UNAUTRE}

Comme son nom l'indique, l'Association desfamilles des détenus-disparus ${ }^{7}$ a cette construites sur ce principe : aucune ne s'est ouvertement définie comme association
de victimes ${ }^{8}$. Si victime il y a, c'est d'abord l'autre, la douleur que ses proches peuvent

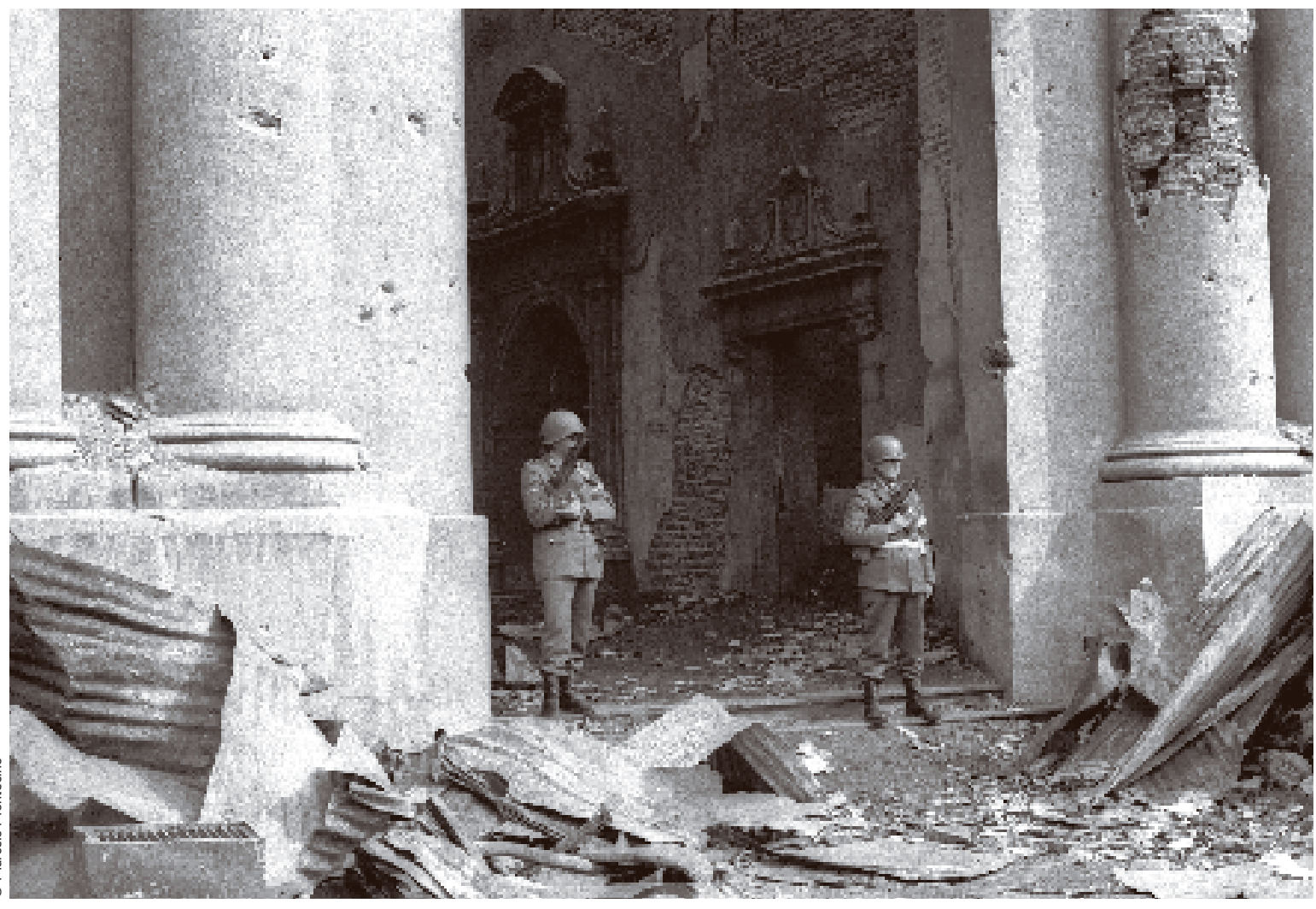

_Coup d’État militaire au

éprouver ne donnant pas droit à ce statut. Le sujet des actions et des discours est le disparu que les vivants représentent. Or, ce cas précis comporte une anomalie : l'impossibilité de qualifier ce crime qui «invisibilise » les sujets recherchés.

En 1973, les familles font fondamentalement face à une situation de méconnaissance. Ce qu'il faut bien comprendre, c'est que le terme de « disparition » n’a de sens que parce qu'il existe un «en dehors» des centres clandestins de détention. À l'intérieur, il n'y a pas de « disparus ». Dans ce sens précis où les prisonniers ne disparaissent pas à leurs propres yeux, pas plus qu'ils ne disparaissent aux yeux de leurs bourreaux aussi longtemps qu'ils sont prisonniers.

Face à cette situation, le travail des familles connaît plusieurs étapes. Tout d'abord, elles chercheront des prisonniers qu'elles espèrent retrouver en vie. Peu à peu interviendra l'idée de la mort, puis la certitude qu'elles ne retrouveront que des dépouilles si tant est qu'on puisse les retrouver ${ }^{9}$ D'un stade à l'autre, les actions se précisent et se diversifent Tout comme les discours qui vont se structurer autour précisent seur

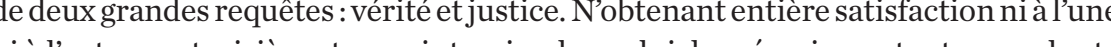
ni à l'autre un troisième terme interviendra, celui de mémoire en tant que volonté présidentiel de La Moneda
après les bombardements du 11 septembre 1973

(9) II semblerait quau Chili, II

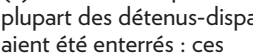
inhumations illégales ont parfois été réalisées dans des cimetières sous les lettres $\mathrm{NN}$ (nomention
nescio). Mais par definition. une personne disparue peut être enterrée pratiauement également des cas dinninération et dautres où des corps ont êté 
CHILI
Quand la victime Quand la victim
est introuvable est intro
(suite)

de ne pas abandonner les êtres chers à leur sort et d'en assurer une présence dans la société.

On peut à grands traits identifier deux espaces où évolue l'AFDD. Celui où sont menées des tâches administratives et celui qui est tourné vers un autre public. Le premier regroupe les initiatives destinées à promouvoir toute démarche légale susceptible d’aider à déterminer ce qui est arrivé. Le second se développe en parallèle, surtout à partir de l'année 1977, et consiste dans des grèves de la faim, manifestations, fabrication dobjets destinés à mettre en images eten mots ceque les familles

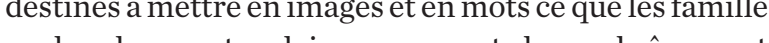
dénoncent. Pantiles aux grilles du Congès : les manifestations étant interdises le manifestantes s'enchainaient pour que la police ne puisse pas les arrêter sur-lechamps. Cette tactique permettait de gagner du temps, de semer un maximum de désordre pour dire ce qu'elles avaient à dire aux passants, mais aussi pour mettre en évidence la violence que les autorités avaient choisi de cacher comme si le calme $-\mathrm{Le} \ll \mathrm{No}+»$ était revenu au Chili.

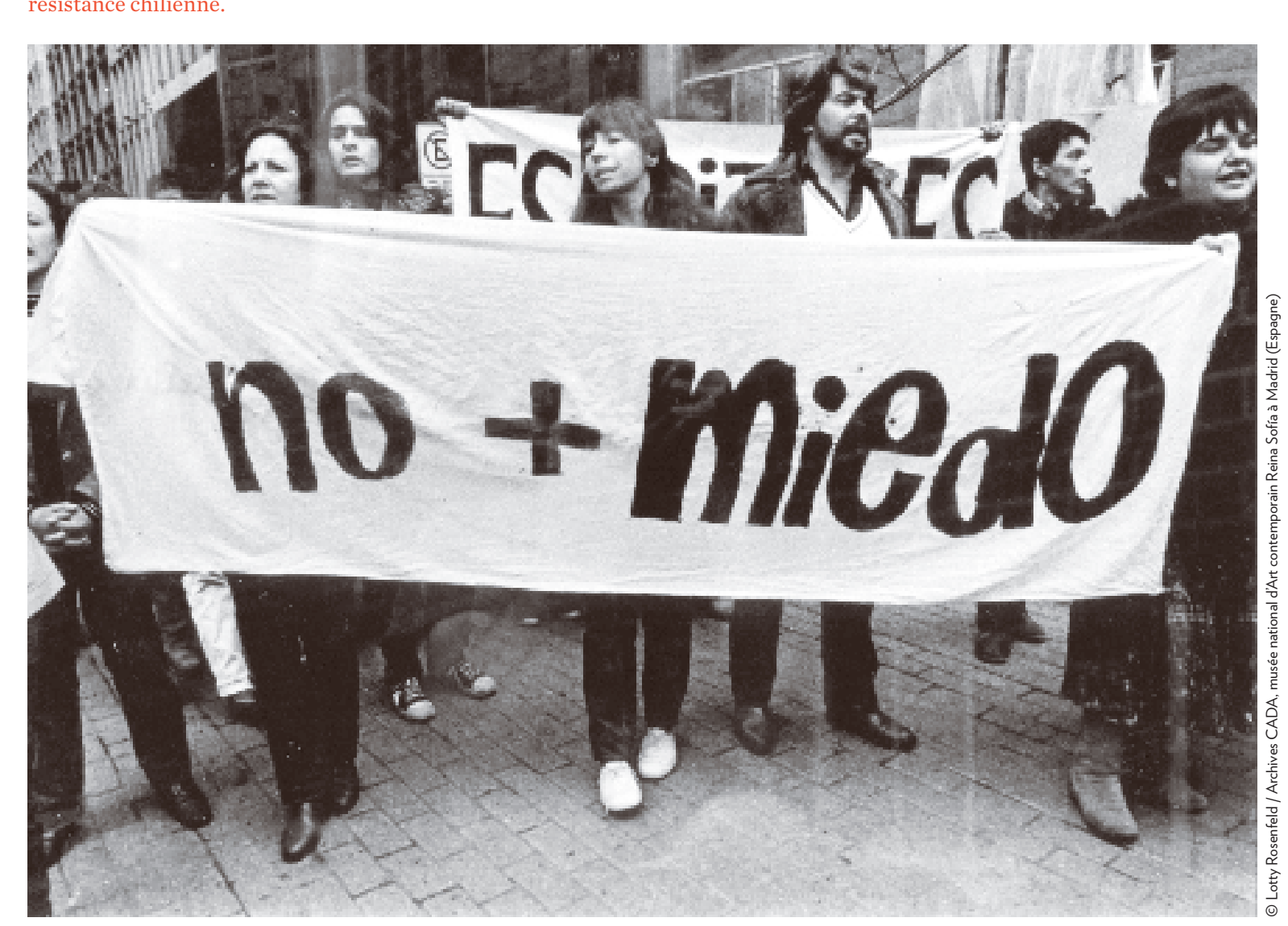

Il s'agissait en tout état de cause de s'informer sur une situation niée officiellement et de la rendre visible. Mais aussi de négocier, au quotidien, avec soi-même et les autres, la place du singulier et du collectif. À cet égard, les pancartes utilisées pendant les manifestations demeurent emblématiques de la posture des membres de l'AFDD. D'assez grandes dimensions, celles-ci donnaient à voir des photographies d'individus recherchés et une seule question pour tous : « Où sont-ils? ? Tel était le pacte : il faut chercher tous les « disparus », car chacun est lêtre cher de quelqu'un. Rarement, la dimension affective d'une histoire politique aura joú un rôle aussi

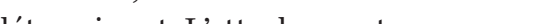
recherchaient leur tenait lieu de raison d'agir et de mode d'exposition ainsi que le montre ce discours prononcé en 1991 :

Nous les aimons parce qu'ils étaient ce qu'ils étaient: libres dans leur manière de penser et justes dans leurs déterminations. Nous les aimons parce qưils étaient des dirigeants de partis politiques populaires, des dirigeants syndicaux, des professionnels motivés par le changement et par la vie. Les raisons que nous avons de les aimer sont exactement celles que d'autres ont eues pour les faire disparaître. Dix-sept ans après ce crime de lèse humanité leur absence nous fait mal comme au premier jour

Cette vaste entreprise d'enquête, de dénonciation et de prise de parole menée par l'AFDD a été conçue comme lutte politique. C'est en tant que représentantes d'acteurs politiques que les mères, les épouses, les sœurs des prisonniers « disparus" ont investi l'espace public tout en travaillant parallèlement, comme dans l'ombre, à des dénonciations de crime en bonne et due forme.

\section{CE CRIME QUE « JE » RACONTE EN LABSENCE DE LA VICTIME}

L’une des principales actions menées conjointement par l'AFDD et le département juridique du Vicariat de la solidarité a été l'ouverture de dossiers où ont été enregistrées les informations relatives aux individus dits détenus-disparus.

Ces dossiers sont nominaux. Ils étaient principalement destinés à motiver des actions de défense du prisonnier, puis, le temps passant, à instruire de futures enquêtesjudiciaires. Ces documents ont joué un rôle important lorsque des enquêtes se sont ouvertes, en particulier après 1989, mais précisons que même lorsque des procès auront lieu, le crime dénoncé ne sera pas la disparition forcée : ce crime n’étant pas codifié, les chefs d'inculpation seront « autres » (exécutions arbitraires, enlèvements aggravés).

Il existe deux grands types de documents: les dossiers informatifs (carpetas) qui contiennent des fiches sur la «victime » et des témoignages sur l'arrestation et, le cas échéant, surla détention ${ }^{11}$; puis les dossiers d'instruction (expedientes) lorsqu'il yaeu intervention de la justice etque des enquêtes sonten cours. Ce matériel d'archive interventive aujourd'hui en partie ouvert aup
(10) Discours prononcé en 1991 lassociation depuis sa création jusqu’à sa mort en 1999. Archive (11) Une partie de ce matériel de Santiago, CDónde estan? (7 volumes), Santiago, Vicaría de

(12) Cf. http: //www. 

autant de docum de naissance, des certificats de mariage, des certificats de scolarité var par leur diversité même, et par leur statut officiel, pouà ces dossiers de

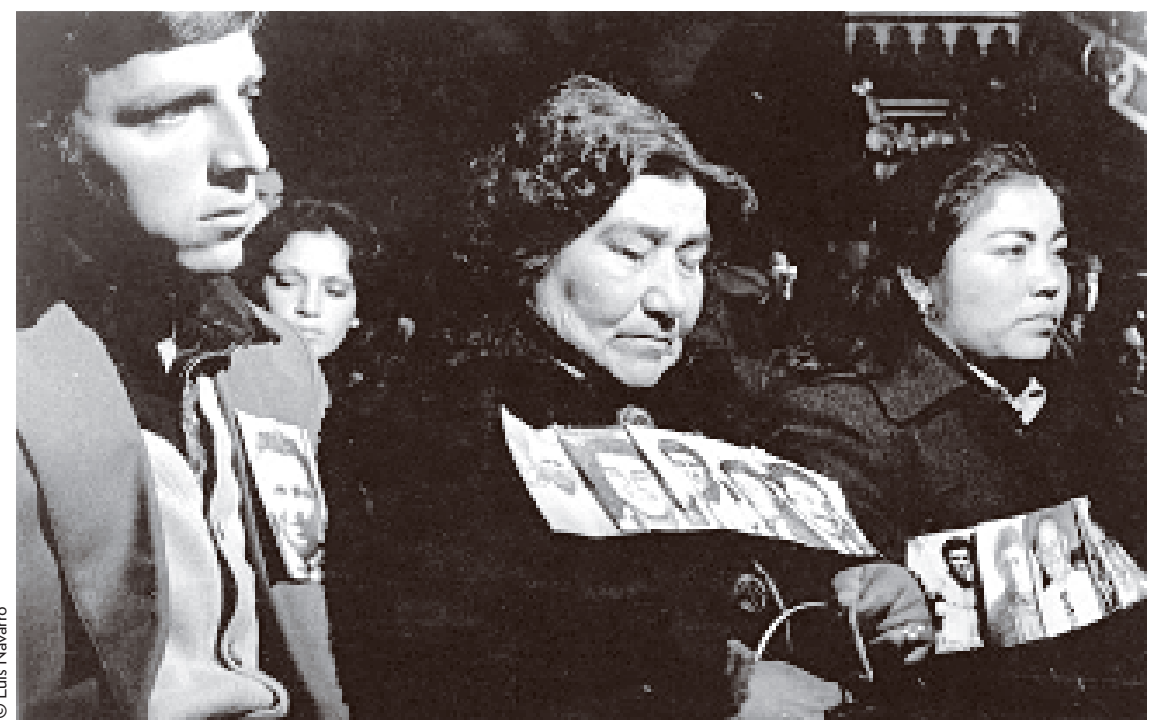
Messe en l'honneur des
détenus-disparus à Santiago détenus-droches dé Recoleta Franciscana 15 septembre 1979 .

partie fondamentale des dossiers : celle où l'on inscrit toute information susceptible de faciliter le travail d'identification des dépouilles. Ainsi, on signale toute caractéristique physique significative. La mémoire des familles devient alors cruciale. Elle est aussi ce qui rend possible une dénonciation, non seulement parce que celle-ci suppose une « loyauté » envers la personne aimée, mais plus prosaïquement parce qu'il s'agit de produire de nombreux détails que seul un être très proche est susceptible de connaître.

Tous ces éléments permettent de comprendre qu’en tant que narration, la disparition est toujours un crime raconté par un autre. Il n'y a pas, au Chili, de « disparu » capable de parler en son nom. Compte tenu de ce que l'on sait aujourd'hui, les « disparus", au Chili sont ceux qui ne sont jamais revenus. Ils ne parlent pas . Ils ne racontent aucune histoire. Ils ne se posent pas en victimes. Ils n'ont aucus! revendication. Sialors qu'ils étaient prisonniers, ils ont eu le temps d'identifier leur situation et de comprendre qu'ils avaient « disparu » aux yeux du monde extérieur, ils n'ont probablement pas songé à utiliser ce terme pour se nommer eux-mêmes. C'est un fait. Quand ils pouvaient encore parler, ces personnes utilisaient d'autres mots pour décliner leurs identités. Et l'on comprend bien, de ce point de vue, la pudeur qu'il peut y avoir au sein des familles : la volonté de ne jamais se substituer à la victime directe, en se postulant elles-mêmes comme victimes.

\section{LAVẼNEMENT DU STATUT DE “VICTIME » ET SES DILEMMES}

À moins qu’elles ne se sentent personnellement et directement lésées. Ce sentiment, relativement absent de la plupart des discours publics de l'association, peut ponctuellement apparaître dans des entretiens réalisés par divers professionnels, 
CHILI
Quand la victime Quand la victime
est introuvable
(suite) (14) Cf notamment les travaux
d'Elizabeth Lira dont Subjetividad
y Pollitica, Santiago, ILAS, 1997. (15) Lorena Pizarro, citée le
5 décembre 2011 par Radio
Coperativa notamment des psychologues, ayant enquêté sur les familles. Il s'agit donc d'entretiens qui ne sont pas destinés à être traités comme « voix officielle» de l'association. Ces documents indiquent qu'il n'y a pas de dénégation : les familles ne refusent pas de se considérer elles-mêmes comme victimes, mais cela n'est pas une prioritét ${ }^{14}$.

Ce qui va se modifier au cours du temps c'est le type de difficultés auxquelles les familles seront confrontées. Dès le changement de régime, survenu en 1989, elles devront faire face à une situation apparemment contradictoire: d'un côté, le nouveau gouvernement officialise le statut de « victime » et s'adresse à elles comme à des victimes; de l'autre, il refuse d'entendre l'une de leurs principales revendications : victimes; de l'autre, il refuse d'entendre l'une de leuss
la justice. Mais la contradiction n'est qu'apparente.

Ce que ce gouvernement refuse, c'est très précisément de reconnaitre la compo-
constice. Mas la contradiction n'est quappante sante politique non pas tant du crime commis, mais de ce que fut sa dénonciation. La requête de justice est la plus problématique - pour tous les gouvernements démocratiquement élus après 1989 -, car elle implique pour être satisfaite de faire asseoir sur le banc des accusés ceux-là mêmes avec lesquels les nouveaux gouvernants ont pactisé le retour à la démocratie. Actuellement, après de nombreuses tensions et batailles, des procès ont pu voir le jour et des condamnations ont été prononcées. Toutefois, il existe une situation ambiguë liée à un système de réduction de peines qui, dans certains cas, permet de libérer assez rapidement ceux qui ont été reconnus qui, dans certai
coupables.

Concernant la consécration du statut de victime, c'est à l’occasion de la publication du Rapport de la Commission nationale de vérité et justice (ou rapport Rettig) qu'elle a eu lieu. Ce document officialise ce que l'AFDD et le Vicariat avaient énoncé sans avoir le pouvoir de l'imposer comme « vérité ». L'année 1991 marque la fin du soupçon total : ces prisonniers non reconnus pendant la dictature ne sont plus de « soi-disant disparus », mais des « détenus-disparus » victimes d’un crime commi par des agents de l'Etat. Le document fait état de la situation des tués (exécutés politiques) ainsi que de celle des parents. Les familles deviennent alors bénéficiaires d'un ensemble de mesures dites de réparation.

Mais le mal qưon cherche à réparer ne se limite pas à la période dictatoriale. Il peut être le fait de décisions prises pendant la période démocratique. On peut citer l'exemple de l'affaire du Patio 29 concernant des dépouilles exhumées du Cimetière général au début des années 1990, identifiées et rendues à leurs familles. Une expertise effectuée par des étrangers avait assez vite fait état de possibles erreurs. Le gouvernement de l'époque avait décidé d’étouffer l'affaire. Puis, sous le gouvernement de Michelle Bachelet, cela s’était su : des corps avaient été enterrés sous une fausse identité. La présidente a demandé une contre-expertise, mais le mal était fait. On était face à une nouvelle tragédie qui a probablement compromis à jamais toute possibilité pour ces familles de faire le deuil. En 2011, l’Etat a décidé d’indemniser les foyers concernés par ces erreurs d'identification. La présidente de l'Association Lorena Pizarro, s'esta cors déclarée satisfite devant cette decision de l'Association, Lorena Pizarro, s'est alors déclaree satisfaite devant cette décision relative « a ceux qui sont victimes de disparitions forcées, d'exécutions politiques,
et à la douleur que cela signifie ${ }^{15}$.»

\section{INQUALIFIABLE}

On aura compris que l'expression «détenu-disparu » n'émane pas des bourreaux. Ceux-là ont d'autres façons de nommer et de faire. Au Chili, le travail de qualification a d'abord été le fait de l'AFDD et du Vicariat de la solidarité. Ils ont travaille à la production de documents utilisés par la suite dans le cadre d'une politique officielle de qualification des victimes commencée au cours du premier gouvernement démo-

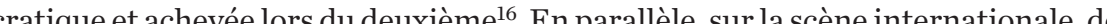
cratique ta nombreuses actions ont été menees par des associations de familles de détenusdisparus de divers pays. Le 23 décembre 2010 est entée en vigueur la Convention internationale pour la protection de toutes les personnes contre les disparitions forcées. Parmi les signataires, l'État chilien.

Ce travail de qualification était nécessaire dans un contexte de mensonge systématique. Mais il n’y a pas eu à choisir. Le terme de « victime » tel qu’utilisé par les parents des détenus-disparus a toujours coexisté avec d'autres. Concrètement, il a permis de mener des actions jugées nécessaires qui n’empêchaient pas lélaboration d'un discours axé sur d'autres modes de présentation de l'autre et de soi-même. La relation familiale, en effet telle qu'elle a été mise en exeroue, a introduit une rationalité nouvelle. En vertu de celle-ci, nous, les citoyens chiliens, avons compris que nalté ces mères, ces épouses, ces filles étaient aussicelles que le pouvoir militaire n'avait pas réussia disss ont parlé de leurs êtres chers nous a amenés à nous poser bien des questions, don celle de la douleur, toujours renouvelée, qui ne saurait faire l'objet d'aucune qualification. Mais aussi la question politique par excellence du pourquoi : Qui l'État a-t-il fait disparaître? Des hommes et des femmes « libres dans leur manière de penser et justes dans leurs déterminations. » 